\title{
DESIGN AND EVALUATION OF \\ DOXYCYCLINE/COLLAGEN/CHONDROITIN SULFATE DELIVERY SYSTEMS USED FOR CARTILAGE REGENERATION
}

\author{
MARIA-MINODORA MARIN ${ }^{1,2}$, MĂDĂLINA GEORGIANA ALBU KAYA ${ }^{2 *}$, MIHAELA \\ VIOLETA GHICA ${ }^{3}$, ELENA DĂNILA ${ }^{1}$, GHEORGHE COARA ${ }^{2}$, LĂCRĂMIOARA POPA ${ }^{3}$, \\ CIPRIAN CHELARU ${ }^{2}$, DURMUȘ ALPASLAN KAYA ${ }^{4}$, VALENTINA ANUȚA ${ }^{3}$, \\ CRISTINA-ELENA DINU-PÎRVU ${ }^{3}$, IOAN CRISTESCU ${ }^{5}$ \\ ${ }^{1}$ University Politehnica of Bucharest, Faculty of Applied Chemistry and Materials Science, 1-7 \\ Gheorghe Polizu Str., 011061, Bucharest, Romania \\ ${ }^{2}$ National Research and Development Institute for Textile and Leather, Division Leather and \\ Footwear Research Institute, Collagen Department, 93 Ion Minulescu Str., 031215, Bucharest, \\ Romania; albu_mada@yahoo.com \\ 3 "Carol Davila" University of Medicine and Pharmacy, Faculty of Pharmacy, Physical and \\ Colloidal Chemistry Department, 6 Traian Vuia Str., 020956, Bucharest, Romania, \\ ${ }^{4}$ Mustafa Kemal University, Faculty of Agriculture, Department of Field Crops, 31034, Antakya- \\ Hatay, Turkey \\ ${ }^{5}$ Carol Davila" University of Medicine and Pharmacy, Faculty of Medicine, Department of \\ Orthopaedy, 8 Bulevardul Eroilor Sanitari, 050474, Bucharest, Romania
}

\begin{abstract}
Cartilage damage is difficult to self-heal due to an avascular microenvironment and distinct mechanical properties. These features are a challenge in designing a cartilaginous tissue with repairing effect without producing any local infections. Thus, a biodegradable scaffold in which the drug can be incorporated is preferable. Drug delivery systems based on collagen sponges have progressively become remarkable biomaterials for different medical applications. The aim of this work was to design and characterize some collagen/chondroitin sulfate supports with doxycycline for cartilage tissue regeneration. The doxycycline should prevent the development of potential infections. Collagen, chondroitin sulfate and doxycycline gels were cross-linked with different concentrations of glutaraldehyde and then freeze-dried in order to obtain collagen matrices. The structural characteristics for the new synthesized biomaterials were firstly assessed by infrared spectroscopy (FT-IR), and scaffolds morphology was then evaluated by optical microscopy and water uptake. The enzymatic biodegradation was also performed. Also, the sponges surface properties were quantified through contact angle. The in vitro doxycycline kinetics release was performed with a dissolution equipment and the release mechanism was investigated. The obtained results recommend these new scaffolds based on doxycycline/collagen/chondroitin sulfate as a promising approach for the treatment of cartilage problems.
\end{abstract}

Keywords: collagen, doxycycline, chondroitin sulfate

\section{INTRODUCTION}

The cartilage tissue is powerless to self-repair and restore due to the absence of nerves, blood vessels and lymphatic tissues, which makes the treatment more difficult (Huang et al., 2016). These features are a challenge in designing a cartilaginous tissue with repairing effect without producing any local infections. Thus, a biodegradable scaffold in which the drug can be incorporated is preferable (Song et al., 2017; Huey et al., 2012). Collagen, a natural biomaterial, represents the main protein from the human organism: skin, bone, cartilage, organs, blood vessels, ligaments and tendons (Miao et al., 2018). Collagen exhibits excellent properties, such as low antigenicity, gradual biodegradability, good biocompatibility and optimal cell proliferation (Lan et al., 2019). Drug delivery systems based on collagen sponges have progressively become remarkable biomaterials for different medical applications (Vikash et al., 2013). 
Chondroitin sulfate (CS), a fundamental component of extracellular matrix in conjunctive tissue (cartilage, bones etc.), is a glycosaminoglycan which helps the formation of aggrecans in the native cartilage (Bang et al., 2018). Collagen and chondroitin sulfate can be great candidates for the design of cartilage tissue engineering in controlling cellular performances (Zhang et al., 2011; Cao et al., 2008). Doxycycline hyclate $(\mathrm{DH})$ represents a tetracycline antibiotic with a strong broad-spectrum activity against aerobic microorganisms and it is usually used in the treatment of the infections (Raval et al., 2014). Thus, the aim of this work was to design and characterize some collagen/chondroitin sulfate supports with doxycycline, for cartilage tissue regeneration.

\section{MATERIALS AND METHODS}

\section{Materials and Preparation of the Scaffolds}

The type II collagen was extracted from bovine cartilage using technology currently available at the National Research and Development Institute for Textile and Leather, Division Leather and Footwear Research Institute - Collagen Department. Doxycycline hyclate and chondroitin sulfate were purchased from Sigma-Aldrich, China. Sodium hydroxide and hydrochloric acid were of analytical grade. Type I collagenase obtained from Clostridium histolyticum was purchased from Sigma-Aldrich, Germany and glutaraldehyde (GA) from Merck, Germany.

The concentration of each collagen gel was adjusted at $1.5 \%$ and $7.4 \mathrm{pH}$ using $1 \mathrm{M}$ sodium hydroxide. $0.2 \% \mathrm{DH}$ and different concentration of CS were added to collagen gel $(\mathrm{w} / \mathrm{v})$, and then the collagen gels were cross-linked with $0.0025 \%$ GA as Table 1 presents. The samples were coded as follows: $\mathrm{M}-1 \div \mathrm{M}-5$.

Table 1. Composition of the obtained samples

\begin{tabular}{ccc}
\hline Sample & Doxy, $\%$ & CS, $\%$ \\
\hline M-1 & 0 & 0 \\
M-2 & 0.2 & 0.05 \\
M-3 & 0.2 & 0.10 \\
M-4 & 0.2 & 0.15 \\
M-5 & 0 & 0.05 \\
\hline
\end{tabular}

\section{FT-IR Analysis}

FT-IR spectral measurements were recorded by a Jasco FT-IR 4200 spectrophotometer. All the spectra were recorded at the following parameters: spectral range $4000-600 \mathrm{~cm}^{-1}$, resolution $4 \mathrm{~cm}^{-1}$ with 30 acquisitions per each sample.

\section{Optical Microscopy Analysis}

The morphology of the designed sponges was carried out using a LEICA optical microscope model S8AP0, with increase power of 20-160x.

\section{Contact Angle Evaluation}

The sponges surface wettability was assessed with CAM 101 (KSV Instruments), using the pendant drop dynamic method, as reported in our previous works (Ghica et al., 2013; Popa et al., 2013). 


\section{Water Uptake}

In order to evaluate the water absorption, the obtained samples were immersed in ultrapure water. At timetabled intervals, the samples were weighed. The experiment was done in triplicate. The water uptake was calculated using the equation: $\%$ Water uptake $=\frac{W_{t}-W_{d}}{W_{d}} \times 100$

where $\mathrm{W}_{\mathrm{d}}$ is the weight of the dry samples and $\mathrm{W}_{\mathrm{t}}$ is the weight of the swollen sample at immersion time $\mathrm{t}$.

\section{Degradation Studies}

Enzymatic degradation of sponges was investigated by measuring the weight loss depending on contact time to collagenase solution. At specific intervals, the swollen scaffolds were weighed. The percentage of scaffold degradation was determined by the following equation:

$$
\% \text { Weight loss }=\frac{W_{i}-W_{t}}{W_{t}} \times 100
$$

where $\mathrm{W}_{\mathrm{i}}$ is the initial weight and $\mathrm{W}_{\mathrm{t}}$ is the weight after time $\mathrm{t}$.

\section{Drug Release Kinetics Analysis}

In vitro drug release from collagen spongious matrices was carried out using a "sandwich" device adapted to a paddle dissolution equipment, as described in our previous studies (Albu et al., 2010). The concentration of doxycycline hyclate released in the medium (phosphate buffer $\mathrm{pH}$ 7.4) at different time intervals was monitored by UV spectroscopy and the cumulative released drug percentage was evaluated.

\section{RESULTS AND DISCUSSION}

The collagen gels with compositions according to Table 1 were freeze-dried and spongious matrices were obtained and characterized.

From the FT-IR spectra (Figure 1) the characteristic bands from collagen it can be observed: amide A, B, I, II and III for all the samples (Albu, 2011).

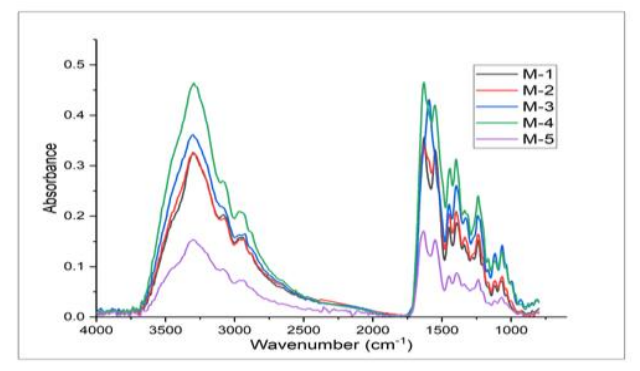

Figure 1. FT-IR spectra
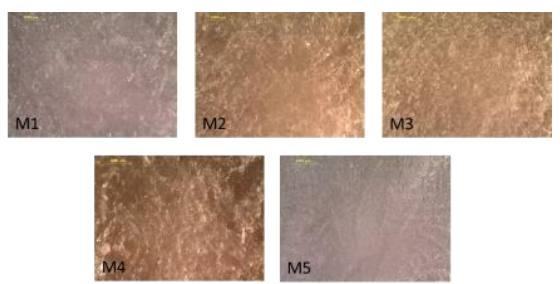

Figure 2. Optical microscopy (20X)

There are no significant changes when doxycycline hyclate and different concentration of chondroitin sulfate were added to collagen gel.

The optical results for the obtained samples are present in Figure 2. Optical images have shown for all the samples a highly porous structure with interconnected pores presenting various dimensions and shapes which is beneficial to the cell adhesion and proliferation.

https://doi.org/10.24264/icams-2020.II.16 


\section{Design and Evaluation of Doxycycline/Collagen/Chondroitin Sulfate Delivery Systems Used for Cartilage Regeneration}

The spongious matrices surface wettability was expressed through contact angle $\left(\mathrm{CA}^{\circ}\right)$ value. The drop shape (Figure 3$)$, monitored with a digital camera, was mathematically described by the Young equation (eq. 3):

$\gamma_{S G}=\gamma_{S L}+\gamma_{L G} \cdot \cos \theta$

where $\gamma_{S G}$ is the interfacial tension S/G, $\gamma_{S L}-$ the interfacial tension S/L, $\gamma_{L G}-$ the superficial tension $\mathrm{L} / \mathrm{G}$ and $\theta-$ the contact angle.

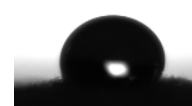

a) $89.03^{\circ} \pm 2.01$

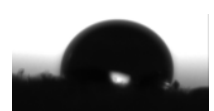

b) $69.58^{\circ} \pm 1.06$

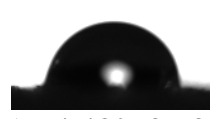

c) $74.48^{\circ} \pm 0.60$

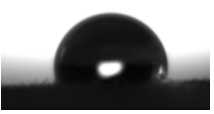

d) $82.24^{\circ} \pm 2.49$

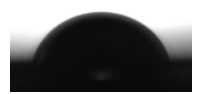

e) $55.64^{\circ} \pm 2.85$

Figure 3. Images of the drop shape for the spongious matrices and the corresponding contact angle values: a) M-1; b) M-2; c) M-3; d) M-4; e) M-5

As it can be noticed in Figure 3, the contact angle values are smaller than $90^{\circ}$, with values between 55.64 and $89.03^{\circ}$, indicating an adequate hydrophilicity of the sponges surface and consequently a good wetting ability by the biologic fluids at the application site. The $\mathrm{CA}$ is strongly influenced by the sponges formulation. Thus, the highest value of CA was recorded for the sample M-1 without drug and chondroitin sulfate, and with a high collagen concentration. The addition of CS $(0.05 \%)$ in the sponge formulation (M-5) lead to the smallest CA, the decrease being about 1.6 times. It seems that the CS presence produced a more porous structure of the sponge surface allowing a better wetting. On the other hand, for the spongious matrix with the same concentration of CS $(0.05 \%)$, but with $0.2 \%$ doxycycline hyclate (M-2) an increase of CA about 1.25 times was observed. These results are in line with our previous studies which highlighted that doxycycline has a cross-linking effect (Albu et al., 2009), inducing in this way a decrease of sponge surface hydrophilicity. Concerning the sponges M-2 $\div$ M4 with the same concentration of biopolymer, drug and cross-linking agent, the increase of CS amount determined an increase of CA, more obvious for higher concentration $(0.15 \%)$.

The water uptake for the studied samples is presented as kinetics during 24 hours in Figure 4. In Figure 4 it can be observed the water absorption during a 24 hours period for the studied samples. All the samples exhibited a high water uptake, this being in concordance with contact angle studies. The maximum amount of water was absorbed by M-5 sample which presented the best hydrophilicity.

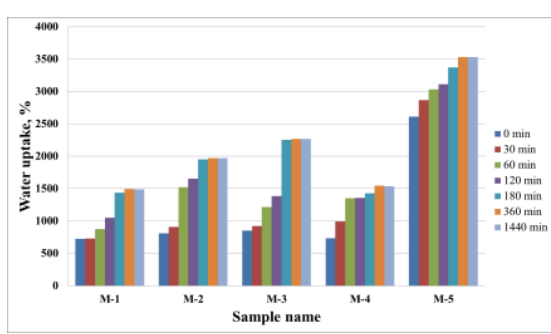

Figure 4. Water uptake during 24 hours for spongious forms

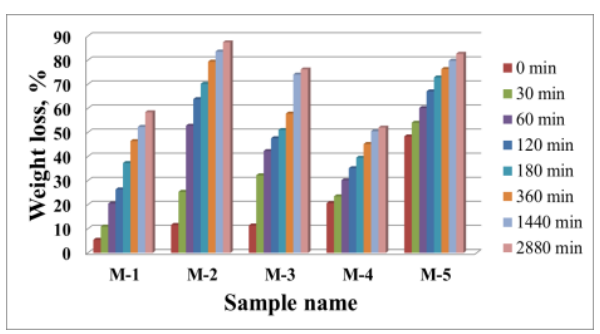

Figure 5. Enzymatic degradation over 48 hours for spongious forms

The degradation results (Figure 5) indicated an excellent biodegradation for all the samples during the $48 \mathrm{~h}$ time interval. The samples with slower degradation rate were M-4 and M-1 with approximately 50\% and 60\% weight loss in 48 hours. 
The in vitro doxycycline kinetic profiles from spongious matrices were recorded as drug cumulative released percentage as a function of time (Figure 6).

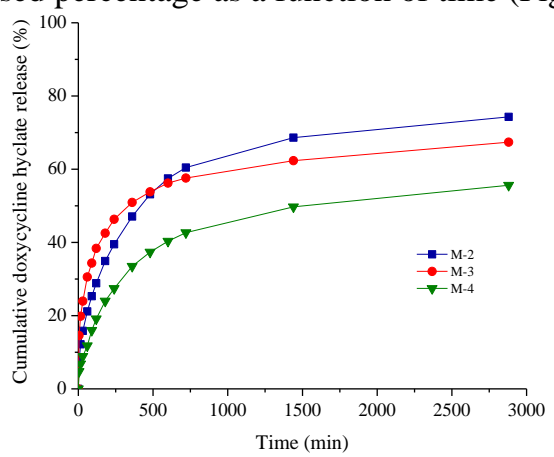

Figure 6. Cumulative release patterns of doxycycline hyclate from collagen spongious matrices as a function of time

The kinetic profiles are similar, with an initial burst release in the first 2 hours, followed by a gradual and prolonged drug delivery over the next 48 hours. The most pronounced rapid release effect was recorded for the M-3 sponge with a medium concentration of chondroitin sulfate $(38.37 \%)$, followed by the M-2 sponge $(28.85 \%)$ with the smallest concentration of CS, while the less evident burst effect was reported for the M-4 sponge (19.13\%). The cumulative doxycycline hyclate released percentage after $48 \mathrm{~h}$ has varied between 55.62 (M-4) and 74.29 (M-2) (Table 2). This long-term antibiotic release provides a local and protective antibacterial effect over a longer period of time necessary for tissue repair.

To set up the drug mass transfer mechanism, the kinetic data were fitted to the Power law model (equation 4 and its particular case, Higuchi model $(n=0.5)$, the corresponding correlation coefficients $(\mathrm{R})$ values being listed in Table 2:

$\frac{m_{t}}{m_{\infty}}=k \cdot t^{n}$

where $m_{t} / m_{\infty}$ represents the fraction of drug released at time $t, k-$ the kinetic constant, $n$ - the release exponent characteristic for the drug release mechanism.

The highest values for the correlation coefficients were recorded for the Power law model, indicating a non-Fickian mechanism for drug release from collagen spongious matrices, in line with our previous studies (Ghica et al., 2013). The release exponent and kinetic constant values specific to the Power law model are given in Table 2.

Table 2. Correlation coefficients for the Power law and Higuchi models; parameter values specific to the Power law model; percentage of drug released

\begin{tabular}{cccccc}
\hline $\begin{array}{c}\text { Spongious } \\
\text { matrices }\end{array}$ & $\begin{array}{c}\text { R Higuchi } \\
\text { model }\end{array}$ & $\begin{array}{c}\mathrm{R} \\
\text { Power Law model }\end{array}$ & $\begin{array}{c}\text { Release } \\
\text { exponent, } \mathrm{n}\end{array}$ & $\begin{array}{c}\text { Kinetic constant, } \\
\mathrm{k}\left(1 / \mathrm{min}^{\mathrm{n}}\right)\end{array}$ & $\begin{array}{c}\text { Drug } \\
\text { Released (\%) }\end{array}$ \\
\hline M-2 & 0.9402 & 0.9806 & 0.31 & 0.069 & 74.29 \\
M-3 & 0.8849 & 0.9850 & 0.22 & 0.133 & 67.38 \\
M-4 & 0.9524 & 0.9801 & 0.34 & 0.039 & 55.62 \\
\hline
\end{tabular}

\section{CONCLUSIONS}

All the designed sponges presented adequate goniometric, morphological and biological properties. The drug release patterns presented a biphasic shape, targeted

https://doi.org/10.24264/icams-2020.II.16 


\section{Design and Evaluation of Doxycycline/Collagen/Chondroitin Sulfate Delivery Systems Used for Cartilage Regeneration}

both to prevent and to control local infection at affected tissue, providing a protective antibacterial effect over the required period to favor long-term healing. The results obtained recommend these new scaffolds based on doxycycline/collagen/chondroitin sulfate as a promising approach for the treatment of cartilage problems.

\section{Acknowledgements}

The work has been funded by the Operational Programme Human Capital of the Ministry of European Funds through the Financial Agreement 51668/09.07.2019, SMIS code 124705 . The study was also financially supported by the Ministry of Research and Innovation, Program 1: Development of National System of Research-Development, Subprogram 1.2: institutional performance projects for funding the excellence in RDI, contract no 6PFE/16.10.2018 and contract no PN 19170302.

\section{REFERENCES}

Albu, M.G. (2011), Collagen Gels and Matrices for Biomedical Applications, Lambert Academic Publishing, Saarbrücken.

Albu, M.G., Ghica, M.V., Leca, M., Popa, L., Borlescu, C., Cremenescu, E., Giurginca, M. and Trandafir, V. (2010), "Doxycycline Delivery from Collagen Matrices Crosslinked with Tannic Acid", Molecular Crystals and Liquid Crystals, 523, 97/[669]-105/[677], https://doi.org/10.1080/15421401003724159.

Albu, M.G., Ghica, M.V., Popa, L., Leca, M. and Trandafir, V. (2009), "Kinetics of In Vitro Release of Doxycycline Hyclate from Collagen Hydrogels", Revue Roumaine de Chimie, 54(5), 373-379.

Bang, S., Jung, U-W. and Noh, I. (2018), "Synthesis and Biocompatibility Characterizations of in Situ Chondroitin Sulfate-Gelatin Hydrogel for Tissue Engineering”, Tissue Engineering and Regenerative Medicine, 15(1), 25-35, https://doi.org/10.1007/s13770-017-0089-3.

Cao, H. and Xu, S.Y. (2008), "EDC/NHS-crosslinked type II collagen-chondroitin sulfate scaffold: characterization and in vitro evaluation", Journal of Materials Science, 19(2), 567-575, https://doi.org/10.1007/s10856-007-3281-5.

Chak, V., Kumar, D. and Visht, S. (2013), A Review on Collagen Based Drug Delivery Systems, Internationa Journal of Pharmacy Teaching \& Practices, 4(4), 811-820.

Ghica, M.V., Albu, M.G., Popa, L. and Moisescu, Ș. (2013), "Response Surface Methodology and Taguch Approach to Assess the Combined Effect of Formulation Factors on Minocycline Delivery from Collagen Sponges", Pharmazie, 68(5), 340-348.

Huang, B.J., Hu, J.C. and Athanasiou, K.A. (2016), "Cell-based tissue engineering strategies used in the clinical repair of articular cartilage", Biomaterials, 98, 1-22, https://doi.org/10.1016/j.biomaterials.2016.04.018.

Huey, D.J., Hu, J.C. and Athanasiou K.A. (2012), "Unlike bone, cartilage regeneration remains elusive", Science, 338, 917-921, https://doi.org/10.1126/science.1222454.

Li, L., Yu, F., Zheng, L., Wang, R., Yan, W., Wang, Z., Xu, J., Wu, J., Shi, D., Zhu, L., Wang, X., Jiang, Q. (2019), "Natural hydrogels for cartilage regeneration: Modification, preparation and application", Journal of Orthopaedic Translation, 17, 26-41, https://doi.org/10.1016/j.jot.2018.09.003.

Miao, Z., Lu, Z., Wu, H., Liu, H., Li, M., Lei, D., Zheng, L. and Zhao, J. (2018), "Collagen, agarose, alginate and matrigel hydrogels as cell substrates for culture of chondrocytes in vitro: a comparative study", Journal of Cellular Biochemistry, 119, 7924-7933, https://doi.org/10.1002/jcb.26411.

Popa, L., Ghica, M.V., Albu, M.G., Orţan, A. and Dinu-Pîrvu, C.E. (2013), "Hysteresis of Contact Angle. Dynamic Wettability Studies of Collagen and Doxycycline Porous Matrices Crosslinked with Tannic Acid", Digest Journal of Nanomaterials and Biostructures, 8(3), 937-943.

Raval, J.P., Naik, D.R., Amin, K.A. and Patel, P.S. (2014), "Controlled-release and antibacterial studies of doxycycline-loaded poly (e-caprolactone) microspheres", Journal of Saudi Chemical Society, 18, 566573, https://doi.org/10.1016/j.jscs.2011.11.004.

Song, X., Zhu, C., Fan, D., Mi, Y., Li, X., Fu, R., Duan, Z., Wang, Y and Feng, R. (2017), "A novel humanlike collagen hydrogel scaffold with porous structure and sponge-like properties", Polymers, 9, 638 https://doi.org/10.3390/polym9120638.

Zhang, L., Li, K.F., Xiao, W.Q., Zheng, L., Xiao, Y.M., Fan, H.S. and Zhang, X.D. (2011), "Preparation of collagen-chondroitin sulfate-hyaluronic acid hybrid hydrogel scaffolds and cell compatibility in vitro", Carbohydrate Polymers, 84(1), 118-125, https://doi.org/10.1016/j.carbpol.2010.11.009. 